\title{
Assessing the Effect of Intrathecal Dexmedetomidine on Cerebrospinal Fluid Levels of Apoptotic Factors: A Clinical Trial Study in Lumbar Disc Surgery
}

\author{
Masoud Nashibi (iD ${ }^{1}$, Parisa Sezari (ii) ${ }^{1}$, Farahd Safari (iD) ${ }^{1}$, Seyyed-Sam-Mahdi Hosaini-Nasab ${ }^{1}$ and \\ Kamran Mottaghi (ii) ${ }^{1, *}$ \\ ${ }^{1}$ Anesthesia Research Center, Shahid Beheshti University of Medical Sciences, Tehran, Iran \\ "Corresponding author: Anesthesia Research Center, Anesthesiology Department, Shahid Beheshti University of Medical Sciences, Tehran, Iran. Mobile: +98-9123804758, \\ Tel/Fax: +98-2155424040, Email: k_mottaghi@sbmu.ac.ir
}

Received 2021 February 07; Accepted 2021 February 16.

\begin{abstract}
Background: Dexmedetomidine protective effects on apoptosis in the brain and peripheral organs have been reported in vivo and in vitro. Apoptotic factors of cerebrospinal fluid (CSF) may influence the prognosis of patients undergoing open discectomy surgery. Objectives: This study evaluated the effect of intrathecal dexmedetomidine administration on the CSF levels of apoptotic factors and clinical outcomes in patients undergoing lumbar discectomy.

Methods: This clinical trial was conducted on patients undergoing open lumbar discectomy. Forty patients were randomly divided into control and dexmedetomidine groups. In the dexmedetomidine group, $0.1 \mu \mathrm{g} / \mathrm{kg}$ of dexmedetomidine was intrathecally injected after anesthesia induction. Patients' hemodynamic status during surgery was recorded; additionally, their pain scores were recorded by the Numeric Rating Scale (NRS) in the recovery room. The levels of apoptotic factors including Bax/Bcl-2 and caspase-3 in the CSF were measured at the beginning and end of discectomy, and the results were compared between the two groups.

Results: Of the 40 evaluated patients, the mean levels of caspase- 3 in the intervention and control groups were $2.28 \pm 0.35$ and $2.34 \pm$ $0.32 \mathrm{ng} / \mathrm{mL}$ before surgery and $2.56 \pm 0.42$ and $2.72 \pm 0.39 \mathrm{ng} / \mathrm{mL}$ after surgery, respectively. The levels of Bax/Bcl-2 in the intervention and control groups were $1.01 \pm 0.11$ and $0.89 \pm 0.07$ before surgery and $1.28 \pm 0.14$ and $1.16 \pm 0.19$ after surgery, respectively. The levels of these two factors were not significantly different. However, the NRS scores were significantly lower in the dexmedetomidine group than in the control group.

Conclusions: Intrathecal dexmedetomidine could significantly and safely reduce the NRS score in the intervention group but did not have any significant effect on the CSF levels of apoptotic factors before and after lumbar discectomy surgery.
\end{abstract}

Keywords: Intrathecal, Dexmedetomidine, CSF, Apoptosis, Apoptotic Factors, Lumbar Spine Surgery, Bax/Bcl-2, Caspase-3

\section{Background}

Lumbar disc herniation is one of the most frequent surgically-treated disorders of the lumbar spine $(1,2)$. However, discectomy-related complications occur in $15-30 \%$ of patients (3-5).

Dexmedetomidine is an alpha 2 adrenergic agonist that is used in the operation theatre and intensive care unit to achieve sedation, analgesia, and anxiolysis. Dexmedetomidine is known for its sedative properties and desired effects in achieving more ventilator-free hours in the intensive care unit (6). Compared to benzodiazepines, it has been proven that intravenous dexmedetomidine prevents delirium among critically ill patients (7). Other favorable characteristics of dexmedetomidine seem to be improved survival among elderly patients and better cognitive function in patients who survive more than three years (8). Moreover, the protective effects of dexmedetomidine on apoptosis and necrosis of the brain and other tissues have been reported (9).

\section{Objectives}

Some of the important apoptotic factors evaluated in this study included Bax/Bcl-2 and caspase-3 in human subjects. The evidence of bio-molecular changes in Bax/Bcl2 and caspase-3 expressions is less clear. Accordingly, this study aimed to evaluate the effects of intrathecal 
dexmedetomidine administration on the CSF levels of apoptotic factors in patients undergoing lumbar discectomy surgery.

\section{Methods}

\subsection{Study Population}

The study population was 20 to 60 years of age people scheduled for lumbar discectomy surgery (Table 1). Of 44 enrolled cases, one case did not have informed consent, two cases were eliminated due to blood transfusion, and one case missed CSF analysis; so, we evaluated 40 cases by the end of the study (Figure 1).

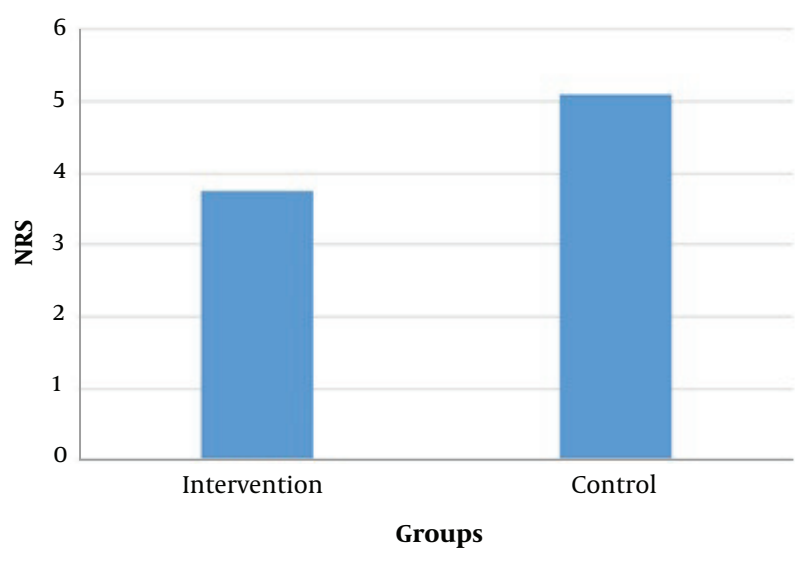

Figure 1. Numerical rating scale scores in two groups

\subsection{Inclusion and Exclusion Criteria}

The inclusion criteria included: (1) patients in the age group of 20 to 60 years who were candidates for lumbar discectomy surgery; (2) no history of receiving antiinflammatory drugs within two weeks before surgery; (3) no immunodeficiency or neurodegenerative diseases; (4) no history of systemic diseases such as diabetes mellitus, renal failure, uremia, hepatic disorders, chronic cardiopulmonary disease, or myopathy; and (5) informed consent. The exclusion criteria included: (1) a change in the approach of surgery; (2) the need for transfusion during surgery; and (3) the duration of surgery of more than four hours.

\subsection{Measurements}

The present study was performed as a clinical trial on patients with lumbar disc herniation who went through discectomy surgery. After obtaining permission from the ethics committee of Shahid Beheshti University of Medical Sciences and selecting the patients to participate in the study, the researchers obtained informed consent. The study subjects were randomly divided into control and dexmedetomidine groups based on the random numbers table. Medical staff and researchers from the beginning of the study closely monitored patients in both groups. The treatment and care protocols were implemented completely and identically in both groups.

A quantitative NRS system was used to assess the level of pain. The induction of anesthesia in all patients was the same using $3 \mu \mathrm{g} / \mathrm{kg}$ of fentanyl, $0.02 \mathrm{mg} / \mathrm{kg}$ of midazolam, and $1.5 \mathrm{mg} / \mathrm{kg}$ of lidocaine as premedication and 2 $\mathrm{mg} / \mathrm{kg}$ of propofol and $0.5 \mathrm{mg} / \mathrm{kg}$ of atracurium as induction agents, and arterial line established to monitor blood pressure. With the patient in a prone position and following the surgical incision and reaching the dura, the surgeon took a one-milliliter sample of the CSF using a 27gauge spinal needle.

The spinal needle in the dexmedetomidine group remained in place, and the syringe was removed. Then, 0.1 $\mu \mathrm{g} / \mathrm{kg}$ of dexmedetomidine was injected into the intrathecal space from the same needle, but in the control group, the needle was removed after sampling. The sampling was performed again at the end of the discectomy, and $1 \mathrm{cc}$ of the CSF was taken again in both groups, according to the reference method. During the surgery, hemodynamic parameters (heart rate and mean arterial pressure) were monitored. In the recovery room, the patients' pain scores were measured by the NRS. After centrifugation at $448 \mathrm{G}$ for 10 minutes, CSF samples were frozen immediately at $70^{\circ} \mathrm{C}$. The collected samples were sent to the laboratory of the medical school, where the apoptotic factors, Bax/Bcl2 and caspase-3 (Zellbio, Germany), were measured by the enzyme-linked immunosorbent assay. The same surgeon performed the entire study process for all patients, including surgery, CSF sampling, and drug injection.

\subsection{Ethical Considerations}

The study group adhered to the principles of medical ethics introduced by the Health Ministry, the Declaration of Helsinki, and legislations in the Medical Ethics Committee of Shahid Beheshti University of Medical Sciences. In addition, the Ethics Committee of Shahid Beheshti University of Medical Sciences approved the protocol of the study. The cost of used medications was supplied by research resources. This investigation did not have any effect on the patients' treatment process, and the result of the assessment was only reported to the patient later. The codes were 


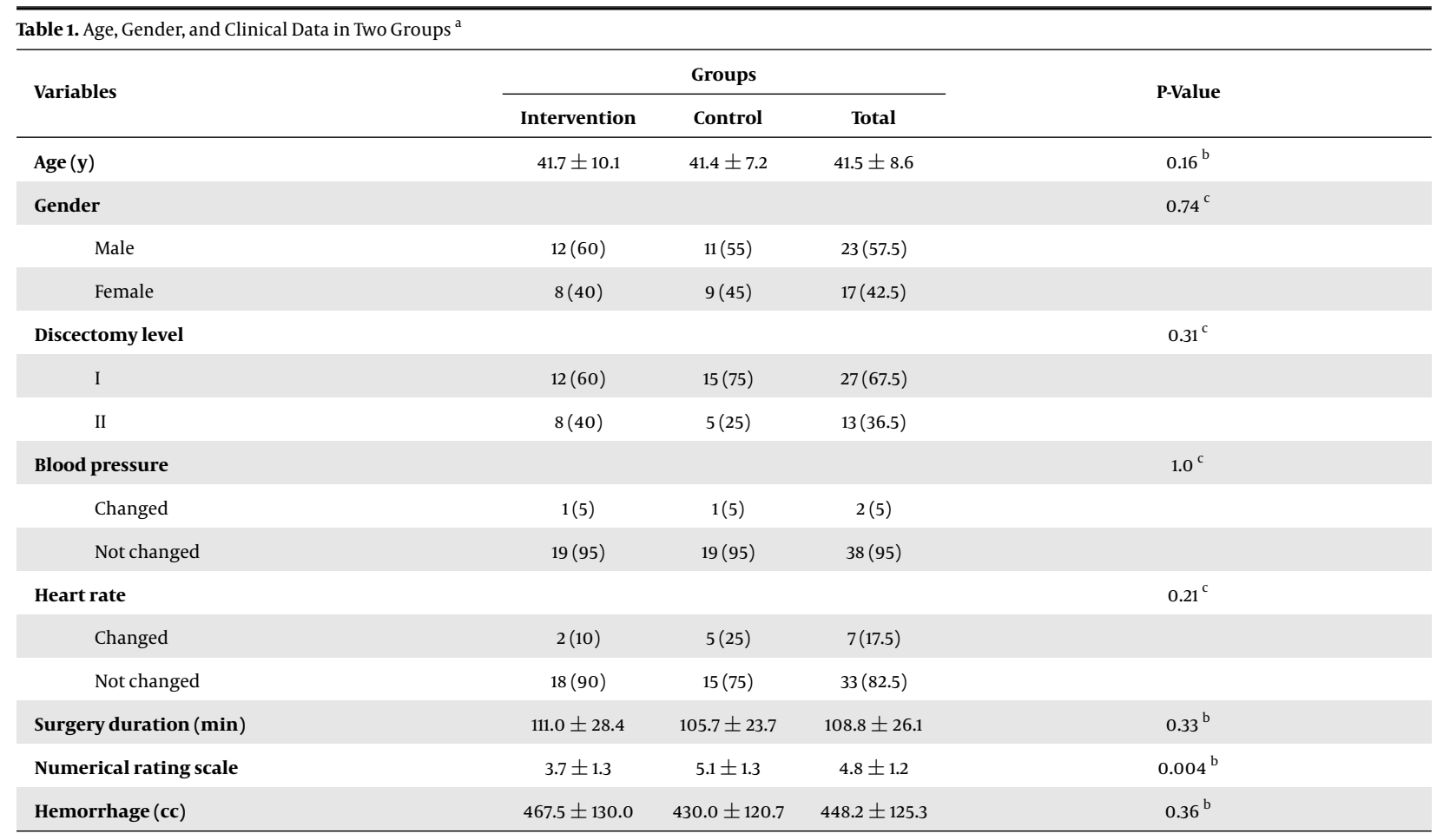

${ }^{\text {a }}$ Values are expressed as mean \pm SD and No. (\%).

${ }^{\mathrm{b}}$ Independent sample $t$ test.

${ }^{c}$ Chi-square test.

as follows: Ethical code, IR.SBMU.MSP.REC.1398.098, IRCT code: IRCT20200127046282N1.

\subsection{Statistical Analysis}

The data were analyzed by SPSS version 23 , and P < 0.05 was considered significant. Means \pm SD were used to present descriptive results of quantitative variables. Ratios and percentages were used to present descriptive results of qualitative variables. Also, a paired t-test and ANOVA test were used for quantitative variable analysis if normality and equality of variances were established, and Wilcoxon and Friedman's tests were used if these test conditions were not met.

\section{Results}

\subsection{Clinical Characteristics}

Demographic data were not statistically different (Table 1). Also, there were no statistically significant differences in clinical data, including discectomy level $(\mathrm{P}=0.31)$, blood pressure change $(\mathrm{P}=1.0)$, heart rate change $(\mathrm{P}=0.21)$, surgery duration $(\mathrm{P}=0.33)$, and hemorrhage volume $(\mathrm{P}=$ 0.36) (Table 1).

\subsection{Numeric Rating Scale}

Using the NRS as a pain scale, the scores of the dexmedetomidine and control groups were $3.7 \pm 1.3$ and 5.1 \pm 1.3 , respectively; based on an independent sample t test, there was a statistically significant difference $(P=0.004)$ (Figure 1).

\subsection{CSF Apoptotic Factors}

The caspase-3 levels in total, intervention, and control groups were $2.31 \pm 0.33,2.28 \pm 0.35$, and $2.34 \pm 0.32 \mathrm{ng} / \mathrm{mL}$ before surgery and $2.64 \pm 0.41,2.56 \pm 0.42$, and $2.72 \pm 0.39$ $\mathrm{ng} / \mathrm{mL}$ after surgery, respectively. In addition, the Bax/Bcl-2 levels in total, intervention, and control groups were 0.95 $\pm 0.09,1.01 \pm 0.11$, and $0.89 \pm 0.07$ before surgery and 1.22 $\pm 0.16,1.28 \pm 0.14$, and $1.16 \pm 0.19$ after surgery, respectively (Table 2).

\subsection{Repeated Measures Test}

Based on a repeated measures test, the CSF levels of caspase-3 $(\mathrm{P}=0.11)$ and Bax/Bcl-2 $(\mathrm{P}=0.95)$ were not significantly different between the intervention and control groups before and after surgery (Figures 2 and 3). 


\begin{tabular}{ccccc}
\hline Table 2. Apoptotic Factors Before and After Disc Herniation Surgery & & & \\
Apoptotic Factors & \multicolumn{2}{c}{ Groups } & Total \\
\cline { 2 - 4 } & Intervention & Control & & Palue (Repeated Measures Test) \\
\hline Caspase-3 (ng/mL) & & & \\
Before & $2.28 \pm 0.35$ & $2.34 \pm 0.32$ & $2.31 \pm 33$ \\
After & $2.56 \pm 0.42$ & $2.72 \pm 0.39$ & $2.64 \pm 0.41$ \\
Bax/Bcl-2 (ratio) & & & \\
Before & $1.01 \pm 0.11$ & $0.89 \pm 0.07$ & $0.95 \pm 0.09$ \\
After & $1.28 \pm 0.14$ & $1.16 \pm 0.19$ & $1.22 \pm 0.16$ \\
\hline
\end{tabular}

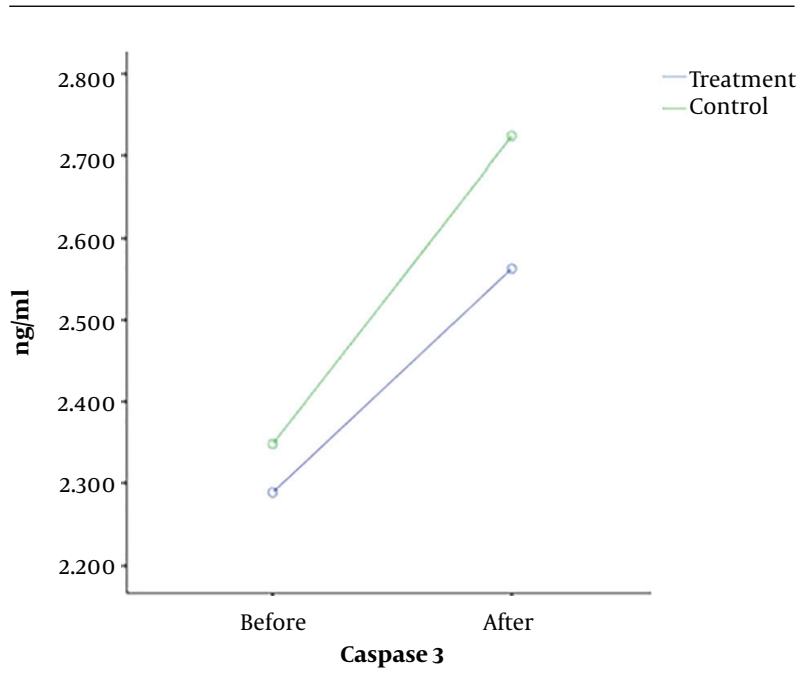

Figure 2. Caspase 3 activity in cerebrospinal fluid before and after surgery

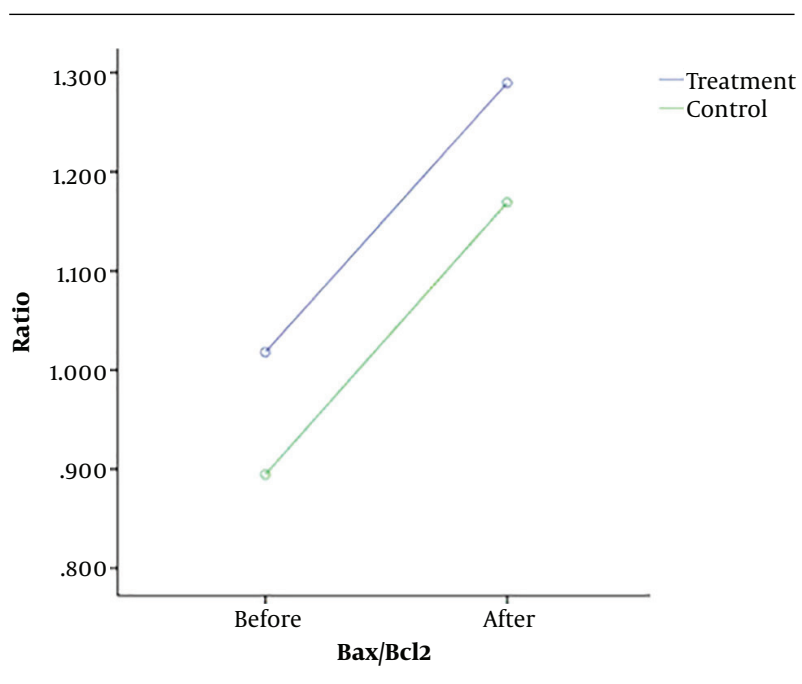

Figure 3. Bax/Bcl-2 activity in cerebrospinal fluid before and after surgery

\section{Discussion}

We observed that the levels of two key apoptotic factors, namely Bax/Bcl-2 and caspase-3, significantly increased in disc herniation surgery, but dexmedetomidine, as an anxiolytic, sedative, and analgesic medication did not have any significant effect on CSF apoptotic factors' levels in our study.

This investigation evaluated the intrathecal dexmedetomidine effect on apoptotic factors in the CSF. Jiang et al. in an observational study on patients with traumatic brain injury (TBI), measured apoptotic factors (caspase-3, sFas, caspase-9, and cytochrome c) in the CSF. They observed that these factors were significantly increased in patients who suffered from TBI (10). Mao et al., in different anesthesia in discectomy, observed that combined spinal-epidural anesthesia and epidural anesthesia caused no significant differences in immune function or inflammatory indices in patients with LDH (MAO) (11). In some other studies, there was a correlation between caspases 1, 3, 6, 7, 8, 9, and 12 and TBI $(12,13)$. In addition, in some studies on other apoptotic factors and TBI, there was a significant enhancement in CSF apoptotic factors (14, 15). Korshunova et al. observed that Bcl-2 could regulate apoptotic factors in myocardial ischemia/reperfusion injury (16). However, these studies were different from our study in terms of protocols. The increased levels of caspase-3 and Bax/Bcl-2 in the CSF of patients shortly after surgery led us to speculate that the surgical intervention may be the sole reason for this increase.

In some studies, the levels of apoptotic factors in brain tissue were measured in patients with cerebral stroke. These studies showed the pathway activation of caspases $1,3,8,9$, and 11, following cerebral ischemia (17); however, Askalan et al. suggested that cell death in infarcts following brain ischemia is partially caspase-3-independent and may also be attributed to nitric oxide (18). Alessandri et al. mentioned that caspase-dependent pathways may also oc- 
cur in an acute subdural hematoma (19). Also, in a study by Montaner et al., they observed that the serum levels of the d-dimer and caspase- 3 combination might be a biochemical marker for faster stroke diagnosis (20). Moreover, in prognosis evaluation, a study by Wang et al. mentioned that CSF caspase-3 enhancement at admission and three days after admission in aneurysmal SAH patients resulted in an unfavorable outcome. In addition, they observed that the levels of these factors were highly associated with injury severity and patients' prognosis after SAH (21). On the same subject, Lorente et al. have recently reported that enhanced serum levels of caspase- 3 are associated with increased mortality in severe TBI cases (22). Accordingly, most studies in this field have indicated that these factors could be prognostic in patients with different neurologic disorders.

\subsection{Conclusion}

We observed that dexmedetomidine as an anxiolytic, sedative, and analgesic medication in anesthesiology, if injected intrathecally, can reduce the NRS in patients undergoing lumbar open discectomy without any significant hemodynamic changes or other side effects, such as excessive sedation. This finding further confirms the earlier investigation results on this subject. Therefore, we recommend intrathecal dexmedetomidine injection for patients undergoing open discectomy as an adjunctive medication for postoperative pain control. Nevertheless, this medication did not significantly affect the CSF levels of apoptotic factors after discectomy. Finally, we believe that assessing the CSF levels of caspase-3 and Bax/Bcl-2 in a more extended postoperative period (i.e., eight, 24, and 48 hours) deserves further investigation.

\section{Footnotes}

Authors' Contribution: Study concept and design, M.N., P.S., F.S., and K.M.; Acquisition of data, S.H. and M.N.; Analysis and interpretation of data, K.M., P.S., and M.N.; Drafting of the manuscript, K.M. and M.N.; Critical revision of the manuscript for important intellectual content, F.S. and P.S.; Statistical analysis, S.H. and P.S.; Administrative, technical, and material support, F.S., M.N., K.M., and P.S.; Study supervision, F.S.

$\begin{array}{lll}\text { Clinical Trial } & \text { Registration } & \text { Code: } \\ \text { IRCT20200127046282N1. } & \end{array}$

Conflict of Interests: The authors declare that they have no conflict of interest.
Ethical Approval: The Ethics Committee of Shahid Beheshti University of Medical Sciences approved the protocol of the study (IR.SBMU.MSP.REC.1398.098).

Funding/Support: The authors declare that they received no funding/support.

\section{References}

1. Ruan W, Feng F, Liu Z, Xie J, Cai L, Ping A. Comparison of percutaneous endoscopic lumbar discectomy versus open lumbar microdiscectomy for lumbar disc herniation: A meta-analysis. Int J Surg. 2016;31:86-92. doi: 10.1016/j.ijsu.2016.05.061. [PubMed: 27260312].

2. Kang Q, Li X, Cheng Z, Liu C. Effects of release and decompression techniques on nerve roots through percutaneous transforaminal endoscopic discectomy on patients with central lumbar disc herniation. Exp TherMed.2017;13(6):2927-33. doi:10.3892/etm.2017.4293. [PubMed: 28587362]. [PubMed Central: PMC5450561].

3. Li X, Han Y, Di Z, Cui J, Pan J, Yang M, et al. Percutaneous endoscopic lumbar discectomy for lumbar disc herniation.J Clin Neurosci. 2016;33:19-27. doi: 10.1016/j.jocn.2016.01.043. [PubMed: 27475315].

4. Kim M, Lee S, Kim HS, Park S, Shim SY, Lim DJ. A comparison of percutaneous endoscopic lumbar discectomy and open lumbar microdiscectomy for lumbar disc herniation in the korean: A meta-analysis. Biomed Res Int. 2018;2018:9073460. doi: 10.1155/2018/9073460. [PubMed: 30175149]. [PubMed Central: PMC6106715].

5. Xu G, Zhang C, Zhu K, Bao Z, Zhou P, Li X. Endoscopic removal of nucleus pulposus of intervertebral disc on lumbar intervertebral disc protrusion and the influence on inflammatory factors and immune function. Exp Ther Med. 2020;19(1):301-7. doi: 10.3892/etm.2019.8223. [PubMed: 31853303]. [PubMed Central: PMC6909559].

6. Reade MC, Eastwood GM, Bellomo R, Bailey M, Bersten A, Cheung B, et al. Effect of dexmedetomidine added to standard care on ventilatorfree time in patients with agitated delirium: A randomized clinical trial.JAMA. 2016;315(14):1460-8. doi: 10.1001/jama.2016.2707. [PubMed: 26975647].

7. Barr J, Fraser GL, Puntillo K, Ely EW, Gelinas C, Dasta JF, et al. Clinical practice guidelines for the management of pain, agitation, and delirium in adult patients in the intensive care unit: Executive summary. Am J Health Syst Pharm. 2013;70(1):53-8. doi: 10.1093/ajhp/70.1.53. [PubMed: 23261901].

8. Zhang DF, Su X, Meng ZT, Li HL, Wang DX, Xue-Ying L, et al. Impact of dexmedetomidine on long-term outcomes after noncardiac surgery in elderly: 3-year follow-up of a randomized controlled trial.Ann Surg. 2019;270(2):356-63. doi: 10.1097/SLA.0000000000002801. [PubMed: 29742525].

9. Kong W, Kang K, Gao Y, Liu H, Meng X, Yang S, et al. Dexmedetomidine alleviates LPS-induced septic cardiomyopathy via the cholinergic anti-inflammatory pathway in mice. Am J Transl Res. 2017;9(11):50407. [PubMed: 29218102]. [PubMed Central: PMC5714788].

10. Jiang W, Jin P, Wei W, Jiang W. Apoptosis in cerebrospinal fluid as outcome predictors in severe traumatic brain injury: An observational study. Medicine. 2020;99(26). e20922. doi: 10.1097/MD.0000000000020922. [PubMed: 32590803]. [PubMed Central: PMC7328954].

11. Mao S, Zhu C, Chang Y. Effects of different anesthesia methods on postoperative transient neurological syndrome in patients with lumbar disc herniation. Exp Ther Med. 2017;14(4):3112-6. doi: 10.3892/etm.2017.4900. [PubMed: 28966685]. [PubMed Central: PMC5613204]. 
12. Zhang X, Alber S, Watkins SC, Kochanek PM, Marion DW, Graham SH, et al. Proteolysis consistent with activation of caspase-7 after severe traumatic brain injury in humans. J Neurotrauma. 2006;23(11):158390. doi: 10.1089/neu.2006.23.1583. [PubMed: 17115905].

13. Darwish RS, Amiridze NS. Detectable levels of cytochrome $C$ and activated caspase-9 in cerebrospinal fluid after human traumatic brain injury. Neurocrit Care. 2010;12(3):337-41. doi: 10.1007/s12028-009-93283. [PubMed: 20087688].

14. Soto-Hernandez JL. Detection of caspase-3, neuron specific enolase, and high-sensitivity C-reactive protein levels in both cerebrospinal fluid and serum of patients after aneurysmal subarachnoid hemorrhage. Neurosurgery. 2008;62(6). E1384. doi: 10.1227/01.neu.0000333328.42706.db. [PubMed:18824967].

15. Au AK, Aneja RK, Bell MJ, Bayir H, Feldman K, Adelson PD, et al. Cerebrospinal fluid levels of high-mobility group box 1 and cytochrome $C$ predict outcome after pediatric traumatic brain injury. $J$ Neurotrauma. 2012;29(11):2013-21. doi: 10.1089/neu.2011.2171. [PubMed: 22540160]. [PubMed Central: PMC3408241].

16. Korshunova AY, Blagonravov ML, Neborak EV, Syatkin SP, Sklifasovskaya AP, Semyatov SM, et al. BCL2-regulated apoptotic process in myocardial-ischemiareperfusion injury (Review). Int J Mol Med. 2021;47(1):23-36. doi: 10.3892/ijmm.2020.4781. [PubMed: 33155658]. [PubMed Central: PMC7723511].

17. Benchoua A, Guegan C, Couriaud C, Hosseini H, Sampaio N, Morin $\mathrm{D}$, et al. Specific caspase pathways are activated in the two stages of cerebral infarction.J Neurosci.2001;21(18):7127-34. [PubMed: 11549723] [PubMed Central: PMC6762989].

18. Askalan R, Deveber G, Ho M, Ma J, Hawkins C. Astrocytic-inducible nitric oxide synthase in the ischemic developing human brain. Pediatr Res. 2006;60(6):687-92. doi: 10.1203/01.pdr.0000246226.89215.a6. [PubMed: 17065568].

19. Alessandri B, Nishioka T, Heimann A, Bullock RM, Kempski O. Caspase-dependent cell death involved in brain damage after acute subdural hematoma in rats. Brain Res. 2006;1111(1):196-202. doi: 10.1016/j.brainres.2006.06.105. [PubMed:16890922].

20. Montaner J, Mendioroz M, Ribo M, Delgado P, Quintana M, Penalba A, et al. A panel of biomarkers including caspase-3 and D-dimer may differentiate acute stroke from stroke-mimicking conditions in the emergency department. J Intern Med. 2011;270(2):166-74. doi: 10.1111/j.1365-2796.2010.02329.x. [PubMed: 21198992].

21. Wang J, Wang JF, Hu XM. Caspase-3 in serum predicts outcome after aneurysmal subarachnoid hemorrhage. Clin Chim Acta.2016;460:196202. doi: 10.1016/j.cca.2016.07.001. [PubMed: 27380999].

22. Lorente L, Martin MM, Gonzalez-Rivero AF, Argueso M, Ramos L, Sole-Violan J, et al. Serum levels of caspase-cleaved cytokeratin18 in patients with severe traumatic brain injury are associated with mortality: A pilot study. PLoS One. 2015;10(3). e0121739. doi: 10.1371/journal.pone.0121739. [PubMed: 25822281]. [PubMed Central: PMC4379106]. 